RELATO DE CASO

ISSN 1677-5090

(C) 2019 Revista de Ciências Médicas e Biológicas

\title{
Manifestações bucais da epidermólise bolhosa: relato de caso
}

\author{
Oral manifestations of epidermolysis bullosa: case report
}

\author{
Renata Portela de Rezende ${ }^{1}$, Naiara Santana Rodrigues², Patricia Miranda Leite Ribeiro ${ }^{3 *}$ \\ ${ }^{1}$ Doutoranda em Processos Interativos dos Órgãos e Sistemas (UFBA). ${ }^{2}$ Residente da Residência Integrada \\ Multiprofissional em Saúde Complexo HUPES (UFBA). ${ }^{3}$ Doutora em Odontologia (UFBA)
}

\begin{abstract}
Resumo
Introdução: a epidermólise bolhosa é uma doença genética rara, que causa a formação de bolhas na pele e na mucosa, por conta de trauma e calor excessivo. Diversas alterações bucais são observadas nesse perfil de indivíduos, como cárie e gengivite devido à dificuldade de higienização. Além disso, devido ao rompimento das bolhas em mucosa, observa-se a formação de úlceras que, ao cicatrizar, podem levar à microstomia, à perda do vestíbulo e à anquiloglossia. Por conta dessas alterações em mucosa bucal, é importante a presença do cirurgião dentista no cuidado desses pacientes, realizando orientações de higiene, assim como tratamento curativo. Objetivo: relatar o caso de uma paciente com diagnóstico de epidermólise bolhosa distrófica recessiva, descrever suas manifestações clínicas bucais e o manejo odontológico. Metodologia: trata-se de um relato de caso de paciente do sexo feminino, com 5 anos de idade, com diagnóstico de epidermólise bolhosa distrófica recessiva. Ao exame clínico, observou-se microstomia, múltiplas lesões ulceradas, gengivite e cáries extensas em diversas unidades dentárias. Foi realizado tratamento das lesões bucais com aplicação de fotobiomodulação a laser, orientações de higiene bucal, profilaxia e controle de biofilme, fluorterapia, além de tratamento das lesões cariosas. Conclusão: o cirurgião dentista tem papel fundamental na equipe multidisciplinar, a fim de promover a saúde bucal desses indivíduos e, consequentemente, melhorar sua qualidade de vida.

Palavras-chave: Epidermólise bolhosa. Higiene oral. Diagnóstico bucal.
\end{abstract}

\begin{abstract}
Introduction: bullous epidermolysis is a rare genetic disease that causes blisters to form on the skin and mucosa due to trauma and excessive heat. Several oral changes are observed in this profile of individuals, such as caries and gingivitis due to the difficulty of hygiene. In addition, due to the rupture of mucosal blisters, ulcers are formed, which, when healing, can lead to microstomy, loss of the vestibule and ankyloglossia. Because of these changes in the oral mucosa, the presence of the dentist in the care of these patients performing hygiene guidelines, as well as curative treatment is important. Objective: to report a case of a patient diagnosed with recessive dystrophic bullous epidermolysis, to describe her oral clinical manifestations and dental management. Methodology: female patient, 5 years old, diagnosed with recessive dystrophic bullous epidermolysis. Clinical examination revealed microstomy, multiple ulcerated lesions, gingivitis and extensive caries in several dental units. Oral lesions were treated with laser photobiomodulation, oral hygiene guidelines, prophylaxis and control of biofilm, fluorotherapy, and carious lesions treatment. Conclusion: the dental surgeon plays a fundamental role in the multidisciplinary team, in order to promote oral health in these individuals and consequently improving their quality of life.

Keywords: Buccal (Oral) Bullous Epidermolysis. Oral Hygiene. Oral Diagnosis
\end{abstract}

\section{INTRODUÇÃO}

A epidermólise bolhosa (EB) é uma doença mucocutânea hereditária, caracterizada pela fragilidade da pele e damucosa, resultando no desenvolvimento de bolhas e que causa alterações sistêmicas variáveis. Os indivíduos afetados apresentam a formação contínua de bolhas, por conta de trauma ou calor e, em alguns casos, as bolhas se desenvolvem sem uma causa aparente (FORTUNA et al., 2015, KUMMER et al., 2013).

A EB foi recentemente descrita em quatro tipos principais, com base no nível de clivagem que ocorre a lesão, sendo elas: EB simples, EB juncional, EB distrófica

Correspondente/Corresponding: *Patrícia Miranda Leite Ribeiro - End: Av. Araújo Pinho, 62 Canela, Salvador - BA, CEP: 40301-155 Faculdade de Odontologia - Tel: (71) 99141-7949 - Email: patrícia.leiteribeiro@ gmail.com e a síndrome de Kindler; e se subdivide de acordo com o padrão de herança genética, morfologia, topografia das lesões e mutação genética envolvida (FINE et al., 2008).

A EB distrófica apresenta-se no padrão genético dominante ou recessivo, onde este último é mais grave e tem maior tendência à formação de bolhas e úlceras (FINE et al., 2008; KUMMER et al., 2013). Ela é caracterizada pela clivagem na sublâmina densa, devido à mutação no gene que codifica o colágeno tipo VII. As manifestações clínicas geralmente ocorrem no nascimento ou durante a infância, com a presença de bolhas generalizadas, onde há envolvimento da mucosa bucal e dentes, unhas, contraturas flexurais das extremidades, estenose anal e uretral, lesões da córnea e anemia devido à má absorção intestinal (BRUCKNER-TUDERMAN, 2010).

Dentre as manifestações bucais da EB, observa-se a presença de úlceras em mucosa, cicatrizes bucais, 
hipoplasia de esmalte, anodontia, anquiloglossia e predisposição maior à cárie devido à dificuldade ao manejo para a higiene bucal do paciente e/ou seu responsável, por provocar-lhe incômodo e desconforto ao mesmo (COHN; TENG, 2016). Em alguns casos, tais lesões podem levar a um fenótipo cicatricial, causando microstomia, anquiloglossia e perda do vestíbulo, provocando severo comprometimento da qualidade de vida e saúde desses indivíduos (FORTUNA et al., 2015).

Desta forma, o tratamento odontológico tem papel fundamental no atendimento multidisciplinar de indivíduos com EB, pois permite a mastigação, favorecendo a nutrição e evita o surgimento de infecções orais e danos ao tecido mole esofágico (GALEOTTI et al., 2014; KRÄMER et al., 2012). Apesar do tratamento sistêmico da EB ainda permaner principalmente paliativo, o cuidado com a saúde bucal pode evitar a destruição e perda da dentição por meio de intervenções e terapia odontológica, mesmo em pacientes afetados mais gravemente (KRÄMER et al., 2012).

O objetivo deste trabalho é relatar o caso de uma paciente com diagnóstico de epidermólise bolhosa distrófica recessiva, descrever suas manifestações clínicas bucais, assim como a importância do cirurgião dentista no atendimento desses indivíduos.

\section{MÉTODO}

Trata-se de relato de caso sobre paciente do sexo feminino, com 5 anos de idade e diagnóstico de epidermólise bolhosa distrófica recessiva, realizando acompanhamento regular em um hospital universitário centro de referência, acompanhada por uma equipe multidisciplinar. A paciente foi encaminhada para o acompanhamento odontológico, devido a uma queixa de odontalgia.

A genitora referiu que as lesões bolhosas surgiram desde o nascimento e que devido à EB a menor já necessitou de internamentos hospitalares (cerca de cinco internamentos) para tratar complicações decorrentes da doença, como anemia por deficiência de ferro, na qual foi necessária a realização de transfusão sanguínea. Em relação ao histórico familiar, na família apenas um indivíduo possui a EB, um primo de primeiro grau. Sobre os hábitos, a genitora relatou que a paciente faz ingestão de dieta pastosa para evitar o trauma e a formação de bolhas na mucosa bucal, sendo esta uma dieta não cariogênica.

No exame físico, a paciente apresentou exulcerações e retalhos de bolhas em dorso superior e membros superiores, crostas melicéricas em membro inferiores e dorso, exsudação purulenta em lesão de membro superior direito e dorso superior/cervical posterior e sinéquia entre ○ 40 e o 5 quirodáctilo da mão esquerda (Figuras 1 e 2).

A paciente usa de Archeus, um ansiolítico homeopático, Tramadol quando está com muitas lesões presentes,
Noripurum para o tratamento da anemia. Além disso, faz uso de curativos especiais nas lesões, utiliza sabonete glicerinado e hidratante corporal. Nega possuir alergias e outras comorbidades.

Figura 1 - Paciente com sinéquia entre 4 e 5 quirodáctilos da mão esquerda

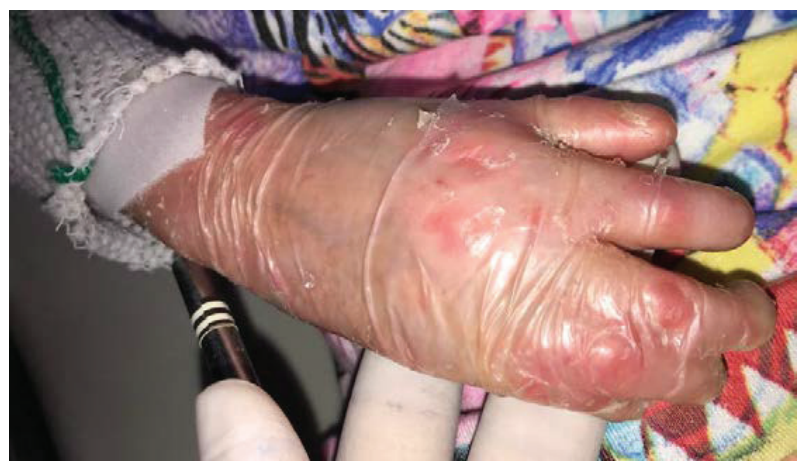

Fonte: As autoras.

Em relação aos hábitos de higiene bucal, é realizada uma vez ao dia pela genitora da paciente, que relata sangramento durante a escovação e não realiza o uso de fio dental, nem enxaguantes bucais. A genitora relata presença constante de bolhas em lábio inferior e de acordo com a escala de dor (0 a 10), a paciente classifica a dor como 7, e que em alguns momentos é superior. De acordo com a genitora, a paciente já apresentou edema na face, associada a uma odontalgia.

Ao exame clínico odontológico, observou-se úlcera em processo de cicatrização no lábio inferior, com aproximadamente $10 \mathrm{~mm}$, úlceras na borda lateral da língua e dorso e na mucosa jugal, além de petéquias dipersas na língua (Figura 2). Foi possível observar uma microstomia (Figura 3). No exame dos dentes, observou-se dentição mista, associada a grande acúmulo de biofilme dentário e presença de eritema e edema gengival, com índice de placa corada de $100 \%$. Observaram-se diversas unidades dentárias com presença de manchas brancas ativas e cáries ativas cavitadas. A paciente relatava dor no primeiro molar decíduo inferior esquerdo (Figura 4).

A paciente, então, iniciou o acompanhamento odontológico ambulatorial, onde foram necessárias sessões de condicionamento do comportamento infantil, visto que a paciente era pouco colaborativa, impossibilitando a realização dos procedimentos odontológicos. Além disso, foram realizadas orientações de higiene bucal, orientando a genitora sobre a importância do cuidado no momento da higienização e mostrando a importância da realização do procedimento. Foi realizada também uma escovação supervisionada com a menor, com a presença da genitora.

Figura 2 - Presença de úlcera em região do lábio inferior e da 
língua

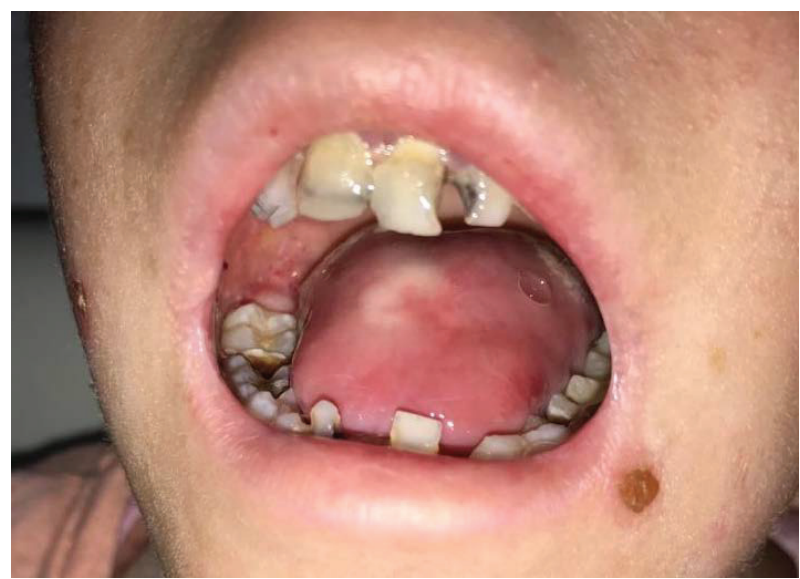

Fonte: As autoras.

Figura 3 - Presença de microstomia, acúmulo de biofilme, inflamação gengival

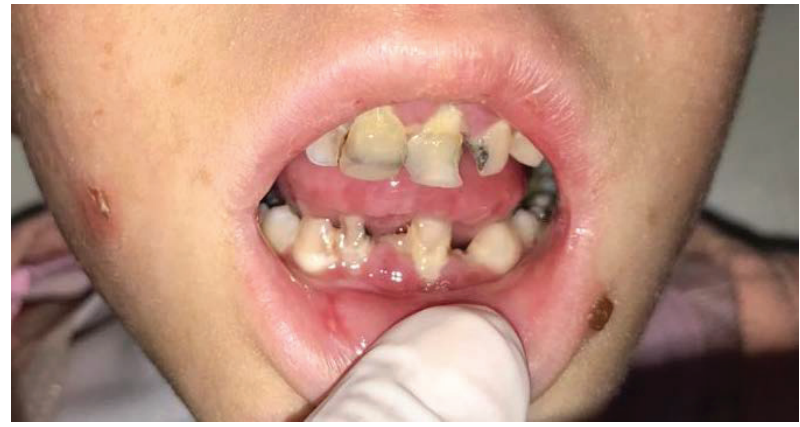

Fonte: As autoras.

Figura 4 - Presença de cáries cavitadas e manchas brancas

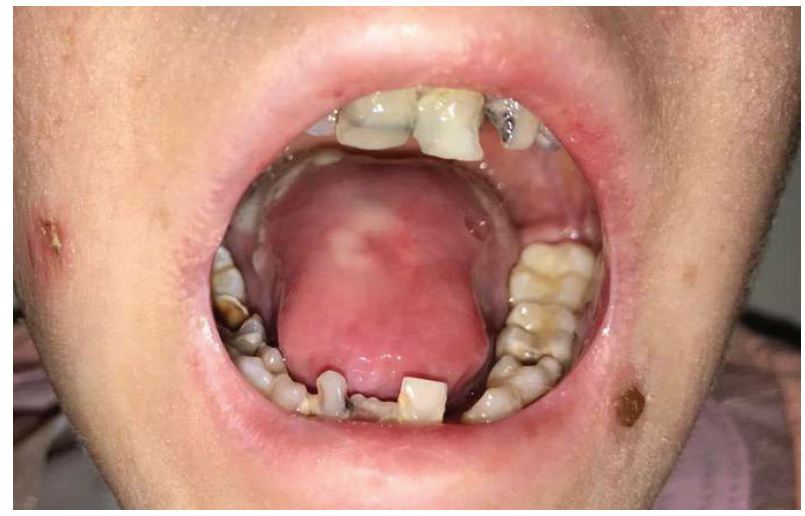

Fonte: As autoras.

Foram realizadas restaurações com cimento de ionômero de vidro nos dentes 74,84 e 85 , sessões de profilaxia bucal e aplicação tópica de flúor, a fim de adequar a cavidade bucal. Por conta da presença de úlceras na mucosa bucal, foram também realizadas sessões de fotobiomodulação com laser vermelho $2 \mathrm{~J} / \mathrm{cm}^{2}$ de forma pontual nas lesões, com o objetivo de auxiliar na cicatrização e na analgesia. Além disso, foi prescrito o uso de clorexidina
0,12\% para auxiliar na higienização bucal. Importante salientar que todos os procedimentos foram realizados de forma cuidadosa na manipulação dos tecidos bucais, utilizando vaselina líquida para o manuseio.

Por conta do difícil manejo da paciente e da dificuldade da genitora em realizar a higienização bucal, ela segue em acompanhamento ambulatorial, para realização de sessões de condicionamento do comportamento infantil, aplicações tópicas de flúor, profilaxia, assim como fotobiomodulação a laser nas lesões ulceradas intrabucais. Além disso, segue em acompanhamento para realização do tratamento curativo, com realização de restaurações nos dentes cariados.

Devido ao fato de a paciente apresentar muita demanda odontológica, não apresentar bom comportamento para a realização do tratamento ambulatorial e ser do interior, tendo dificuldade de acesso ao atendimento, optou-se por concluir os procedimentos restauradores em centro cirúrgico, diminuindo assim o número de consultas necessárias para a realização dos procedimentos.

\section{DISCUSSÃO}

As manifestações sistêmicas da EB comumente estão presentes desde o nascimento. Além de causar a formação de bolhas na pele e na mucosa, o comprometimento das articulações, sistema digestivo, sistema respiratório e fala, também são observados. A EB tem sido associada à desnutrição crônica, por conta da constante formação de bolhas e consequente perda de eletrólitos e proteínas, causando, assim, anemia severa, deficiência de proteína e infecção (FINE et al., 2008; HUBBARD et al., 2011), o que pode ser comprovado no caso em questão, onde a paciente já realizou internamentos hospitalares devido a um quadro de anemia.

Em relação ao caso descrito, observaram-se diversas características encontradas na EB distrófica. As manifestações bucais da EB podem ser observadas tanto em tecidos moles, como em tecidos duros (MELLO et al., 2016), sendo o sinal intrabucal mais comumente encontrado na doença a presença de bolhas intrabucais, como foi observado no caso (MELLO et al., 2016; LINDEMEYER; WADENYA; MAXWELL, 2009). A presença das bolhas compromete a mucosa bucal, causando lesões erosivas dolorosas, dificultando a realização da higiene bucal, consequentemente predispondo à formação de cárie dentária e à inflamação gengival. Outras alterações comumente encontradas na mucosa, em pacientes com EB, são microstomia, perda do fundo de vestíbulo e anquiloglossia, devido ao processo cicatricial das bolhas e ulcerações. Apesar de a paciente não apresentar anquiloglossia e perda do fundo de vestíbulo, observou-se uma diminuição da abertura de boca (KRÄMER et al., 2012; MELLO et al., 2016).

Com o objetivo de auxiliar na cicatrização das úlceras, a fotobiomodulação tem sido uma alternativa indicada para o tratamento das lesões na mucosa bucal. Isso se deve aos seus efeitos bioestimulantes, que auxiliam 
no reparo de feridas e tem efeito analgésico (CAFARO; BROCCOLETTI; ARDUINO, 2012; PARUSHETTI et al., 2013). Foram realizadas sessões de fotobiomodulação a laser na paciente, com o objetivo de reduzir a sintomatologia dolorosa, além de auxiliar no reparo tecidual.

Além das alterações na mucosa, a literatura tem destacado que os pacientes com EB distrófica apresentam alto risco de desenvolver cárie, devido a defeitos de esmalte, higiene bucal inadequada decorrente da presença de bolhas, pela perda da destreza manual e pela xerostomia decorrente do uso de alguns medicamentos. Associado a esse quadro, ocorre também o consumo de uma dieta hipercalórica e, consequentemente, cariogênica, projetada para compensar o quadro metabólico deficiente desses pacientes (AL-ABADI et al., 2016; HUBBARD et al., 2011; KRÄMER et al., 2012; KUMMER et al., 2013). Portanto, tais pacientes devem receber avaliações periódicas para manutenção de saúde bucal, visando a deteç̧ão precoce da doença dentária, a presença de doença periodontal, de biofilme dentário, bem como avaliar a condição salivar e a presença de erosões dentárias (HUBBARD et al., 2011).

A paciente do caso descrito apresentou um alto risco para cárie devido à grande quantidade de biofilme dentário, dificuldade na realização da higiene bucal e, por conta disso, segue em acompanhamento ambulatorial para a manutenção da sua saúde bucal.

O acompanhamento odontológico é fundamental, no entanto, é importante que exista a colaboração do paciente na realização da higienização bucal, a fim de evitar o acúmulo de biofilme. O cuidado com a saúde bucal em pacientes com EB distrófica pode ser difícil devido a limitações graves, como limitação da abertura da boca, formação de bolhas intrabucais e descamação da mucosa, com trauma decorrente de comidas duras e escovação e dificuldade de deglutição imposta pela condição. $O$ desenvolvimento de cicatrizes e contraturas das mãos complica ainda mais os problemas de higiene bucal.

Desta forma, a prevenção é crucial e o cirurgião dentista desempenha um papel central na intervenção precoce (SHARMA; BEDI, 2013). Por isso, a higiene bucal domiciliar deve ser realizada com uma escova dentária com cerdas macias ou, alternativamente, cotonetes, pano de algodão limpo ou gaze, casos a boca esteja muito ulcerada (KRÄMER et al., 2012). Além disso, o uso de clorexidina sem álcool $0,12 \%$ e flúor é fortemente recomendado como terapia adjuvante (FORTUNA et al., 2015; KRÄMER et al., 2012).

Devido às características bucais e dentárias dos pacientes com EB, o tratamento odontológico torna-se desafiador. Por isso, são necessárias várias modificações na realização do tratamento e uma abordagem cuidadosa para evitar o dano tecidual (KRÄMER et al., 2012). O tratamento odontológico em pacientes com EB distrófica requer a lubrificação dos tecidos bucais, luvas e instrumentos para evitar aderência e formação de bolhas. Além disso, a pressão deve ser suave ao manusear o tecido, o sugador deve apoiar-se em tecidos duros para evitar traumas e descamação epiteliais (FORTUNA et al., 2015; GALEOTTI et al., 2014).

O tratamento realizado sob anestesia local muitas vezes é dificultado devido à microstomia e à limitação da abertura da boca, além do risco de formação de boIhas e lesão dos tecidos moles, após administração do anestésico (AL-ABADI et al, 2016). Em muitos casos, a anestesia geral é recomendada quando há necessidade de múltiplos procedimentos cirúrgicos, de tratamento em manifestações graves de EB ou quando o paciente não pode tolerar tratamento odontológico (KRÄMER et al., 2012; AL-ABADI et al., 2016). Diante disso, a paciente foi encaminhada para a realização dos procedimentos restauradores e cirúrgicos em centro cirúrgico, com o objetivo de otimizar o tratamento da mesma.

\section{CONCLUSÕES}

A EB causa diversas alterações na cavidade bucal e, por isso, a prevenção é fundamental para a manutenção da saúde desses pacientes. Desta forma, o tratamento odontológico é parte fundamental nos cuidados multidisciplinares dos pacientes com EB, especialmente nos tipos mais severos, pois a manutenção funcional dos dentes e o cuidado com a higiene bucal reduzem os danos causados pelas lesões, favorecendo a mastigação e a nutrição, melhorando sua qualidade de vida.

\section{REFERÊNCIAS}

AL-ABADI, A. et al. Dental and anaesthetic challenges in a patient with dystrophic epidermolysis bullosa. Sultan Qaboos Univ. Med. J., Oman, v. 16, n. 4, p. e495, 2016.

BRUCKNER-TUDERMAN, L. Dystrophic epidermolysis bullosa: pathogenesis and clinical features. Dermatol clin., Philadelphia, p. 107-114, 2010.

CAFARO, A.; BROCCOLETTI, R.; ARDUINO, P. G. Low-level laser therapy for oral mucous membrane pemphigoid. Lasers med. sci., London, v. 27, n. 6, p. 1247-1250, 2012.

COHN, H. I.; TENG, J. M. C. Advancement in management of epidermolysis bullosa. Curr. opin pediatr., Philadelphia, v. 28, n. 4, p. 507-516, 2016.

FINE, J. D. et al. The classification of inherited epidermolysis bullosa (EB): Report of the Third International Consensus Meeting on Diagnosis and Classification of EB. J. Am. Acad. Dermatol., St. Louis, n. 58, n. 6, p. 931-950, 2008.

FORTUNA, G. et al. Clinical features of gingival lesions in patients with dystrophic epidermolysis bullosa: a cross-sectional study. Austr. Dent. J., Sydney, v. 60, n. 1, p. 18-23, 2015.

GALEOTTI, A. et al. Er: YAG laser dental treatment of patients affected by epidermolysis bullosa. Case Reports in Dentistry, [s.I], v. 2014, 2014.

HUBBARD, L. et al. The challenges of meeting nutritional requirements in children and adults with epidermolysis bullosa: proceedings of a multidisciplinary team study day. Clin. exp. dermatol., Oxford, v. 36, n. 6, p. 579-584, 2011.

KRÄMER, S. M. et al. Oral health care for patients with epidermolysis bullosa - best clinical practice guidelines. Int. j. paediatr. dent., Oxford, v. 22, supl. 1, p. 1-35, Sept. 2012.

KUMMER, R. et al. Oral manifestations and challenges in dental treat- 
ment of epidermolysis bullosa dystrophica. J. Dent. Child., Chicago, v. 80, n. 2, p. 97-100, 2013.

LINDEMEYER, R.; WADENYA, R.; MAXWELL, L. Dental and anaesthetic management of children with dystrophic epidermolysis bullosa. Int. j. paediatr. dent., Oxford, v. 19, n. 2, p. 127-134, 2009.

MELLO, B. Z. F. et al. General anesthesia for dental care management of a patient with epidermolysis bullosa: 24 month follow up. Spec. care dentist., Chicago, v. 36, n. 4, p. 237-240, 2016.

PARUSHETTI, A. D. et al. Oral manifestations of epidermolysis bullosa dystrophica: a rare genetic disease. BMJ Case Reports, v. 2013, p. bcr2012007963, 2013.

SHARMA, S.; BEDI, S. Dystrophic epidermolysis bullosa associated with non-syndromic hypodontia. Indian dermatol. online j., Mumbai, v. 4 , n. 4, p. 296, 2013.

Submetido em:04/11/2019

Aceito em: 11/11/2019 